\title{
Waiting in pain
}

\section{Innovative approaches can give more Australians access to pain management}

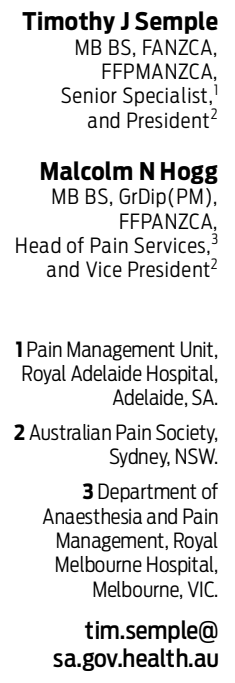

doi: 10.5694/mjal2.10148

Research p 386

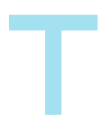
he burden of chronic pain in the Australian community cannot be ignored. The results of the Bettering the Evaluation and Care of Health (BEACH) program suggest that almost $20 \%$ of general practitioner consultations relate to an individual with chronic pain. ${ }^{1}$ This is consistent with the 2006 South Australian Health Omnibus report, with $20 \%$ of adults reporting chronic pain and $5 \%$ reporting pain that interfered extremely with daily activity. ${ }^{2}$ In Britain, the Royal College of General Practitioners (RCGP) notes that severe chronic pain leads to a global health impact equivalent to that of heart disease or severe mental illness. With individuals with chronic pain attending their GPs five times more frequently than those without the condition, the RCGP has accorded chronic pain Clinical Priority status for 2011-2013. ${ }^{3}$

Specialist multidisciplinary pain management services have arisen over the past four decades to support GPs in the care of individuals with chronic pain. The survey by Hogg and colleagues, commissioned by the Australian Pain Society and published in this issue of the Journal, ${ }^{4}$ is the first Australian study to explore the availability of these services. There are long waiting times for an initial appointment at many publicly funded services, with a median wait of 150 days, but several services reported a waiting time of over a year. By comparison, of the $20 \%$ of the population reporting pain, less than $0.2 \%$ will gain access to a specialist service in any given year. For Australians living outside capital cities, the proportion who gain access is even lower.

With the disparity between demand and capacity being so great and waiting lists so long, some GPs choose not to refer at all. Having to wait 6 months for pain management is associated with deterioration of psychological wellbeing and quality of life, ${ }^{5}$ but no referral at all limits the potential options for people with pain. With limited education on pain management in undergraduate curricula, lack of Pharmaceutical Benefits Scheme support for prescriptions of adjuvant non-opioid pharmaceuticals such as gabanoids, and minimal federal medical benefits funding for non-pharmacological therapies for pain management, it can be a very difficult task for time-limited GPs to provide good quality pain management without access to specialist services. Against this background, the difficulties of ensuring appropriate use of prescription opioids in suitable patients and avoiding harms as highlighted recently in the Journal ${ }^{6}$ seems hardly surprising.

Similar shortfalls in the management of chronic pain in the United Kingdom, Canada and the United States have driven a series of health policy initiatives in recent years. In the US, the response has been led by the government- initiated Institutes of Medicine "Relieving Pain in America" blueprint, outlining a comprehensive strategy to tackle the problem. ${ }^{7}$ In the UK, a national pain summit was held in November 2011, and the Canadian Pain Summit is planned for April 2012. Here in Australia, despite the National Pain Summit in 2010 driving the development of the National Pain Strategy ${ }^{8}$ and the formation of Painaustralia (an independent advocacy body supported by the Australian and New Zealand College of Anaesthetists Faculty of Pain Medicine, the Australian Pain Society and leading consumer organisations), the response at a federal health policy level to date has been disappointing.

In response to the unacceptably long waiting lists, innovative approaches have been implemented by two tertiary-based multidisciplinary pain centres. ${ }^{9}$ Recognising that optimising patients' capacity for self-management is a critical but frequently neglected first step, the teams from Fremantle Hospital Pain Medicine Unit in Western Australia and the Hunter Integrated Pain Service in New South Wales have abandoned the existing model of pain service care. By requiring early enrolment in a brief, interprofessional, team-based, group psychoeducational program before individual or team assessment, along with providing web-based resources for consumers for current best evidence in diagnostic and therapeutic approaches, waiting lists have been reduced markedly and throughput of new patients has increased significantly.

The inherent appeal of this low-cost early intervention approach, allied with the demands from both consumers and GPs for improvements in pain management capacity at a community level, has driven one Medicare Local (these are regional organisations for planning and coordinating improvements in primary health care) to incorporate this philosophy ${ }^{10}$ into their service plan. Rolling out similar programs in other Medicare Locals may provide at least part of the solution to the large waiting lists.

The second major driver for change in pain management relates to the negative aspects of escalating prescription opioid use in poorly selected individuals. We believe this issue is intrinsically linked to the deficiencies in providing optimal pain management at a primary care level and impaired access to specialist support services. Real-time opioid prescription monitoring schemes may limit opioid misuse, but regulatory approaches alone will not overcome underlying deficiencies. Regulation needs to be increased, but only in parallel with significantly improved system-wide pain management capacity so that Australians living with pain do not continue to wait in pain. 
Competing interests: No relevant disclosures.

Provenance: Commissioned; externally peer reviewed.

1 SAND abstract No. 150 from the BEACH program 2009-10. Chronic pain in general practice patients. Sydney: AGPSCC University of Sydney, 2010. http:// sydney.edu.au/medicine/fmrc/publications/sand-abstracts/150-Chronic Pain.pdf (accessed Jan 2012).

2 Currow DC, Agar M, Plummer J, et al. Chronic pain in South Australia population levels that interfere extremely with activities of daily living. Aust $N$ Z J Public Health 2010; 34: 232-239.

3 Royal College of General Practitioners. Clinical priorities 2011-2013: Chronic Pain. http://www.rcgp.org.uk/clinical and research/circ/priorities commissioning/clinical_priorities/2011-2013.aspx (accessed Jan 2012).

4 Hogg M, Gibson S, Helou A, et al. Waiting in pain: a systematic investigation into the provision of persistent pain services in Australia. Med J Aust 2012; 196: 386-390.
5 Lynch ME, Campbell F, Clark AJ, et al. A systematic review of the effect of waiting for treatment for chronic pain. Pain 2008; 136: 97-116.

6 Hall WD, Farrell MP. Minimising the misuse of oxycodone and other pharmaceutical opioids in Australia [editorial]. Med J Aust 2011; 195: 248-249.

7 Institutes of Medicine. Relieving pain in America: a blueprint for transforming prevention, care, education, and research. Washington, DC: IOM, 2011. http:// www.iom.edu/Reports/2011/Relieving-Pain-in-America-A-Blueprint-forTransforming-Prevention-Care-Education-Research.aspx (accessed Jan 2012).

8 Painaustralia. National pain strategy: pain management for all Australians. http://www.painaustralia.org.au/strategy/ (accessed Jan 2012).

9 Davies SJ, Hayes C, Quintner JL. System plasticity and integrated care: informed consumers guide clinical reorientation and system reorganization. Pain Med 2011; 12: 4-8. doi: 10.1111/j.1526-4637.2010.01016.x.

10 Davies S, Quintner J, Parsons R, et al. Preclinic group education sessions reduce waiting times and costs at public pain medicine units. Pain Med 2011; 12: 59-71. doi: 10.111/j.1526-4637.2010.01001.x. 